\title{
Predicted Lattice Relaxation Around Point Defects In Zinc Selenide
}

Leonid Muratov

Scott Little

Yaxiang Yang

Bernard R. Cooper

Thomas H. Myers

See next page for additional authors

Follow this and additional works at: https://researchrepository.wvu.edu/faculty_publications

\section{Digital Commons Citation}

Muratov, Leonid; Little, Scott; Yang, Yaxiang; Cooper, Bernard R.; Myers, Thomas H.; and Wills, John M., "Predicted Lattice Relaxation Around Point Defects In Zinc Selenide" (2001). Faculty Scholarship. 638.

https://researchrepository.wvu.edu/faculty_publications/638 
Authors

Leonid Muratov, Scott Little, Yaxiang Yang, Bernard R. Cooper, Thomas H. Myers, and John M. Wills 
PHYSICAL REVIEW B, VOLUME 64, 035206

\title{
Predicted lattice relaxation around point defects in zinc selenide
}

\author{
Leonid Muratov, Scott Little, Yaxiang Yang, Bernard R. Cooper, and Thomas H. Myers \\ Department of Physics, West Virginia University, Morgantown, West Virginia 26506-6315
}

John M. Wills

Theory Division, Los Alamos National Laboratory, Los Alamos, New Mexico 87545

(Received 18 December 2000; published 25 June 2001)

\begin{abstract}
There is great interest in altering the electronic and consequent optical properties of wide-band-gap semiconductors through the use of selected additives (dopants). These effects depend on the way in which the impurity enters the lattice structure, and the resulting possible lattice relaxation effects and their repercussions. There have been several calculations for the point defects in $\mathrm{ZnSe}$ based on pseudopotential approaches, with some differences in results among these; and, in the case of nitrogen substitution for the selenium, there is a difference with experiment as to the expected size of relaxation effects around the defect. Thus it is useful to have calculations by a quite different technique. For that reason, as benchmark calculations, we have studied lattice relaxation around $\mathrm{Zn}$ and $\mathrm{Se}$ vacancies, and around $\mathrm{N}$-for-Se substitution sites in $\mathrm{ZnSe}\left[\mathrm{V}_{S_{e}},\left(\mathrm{~V}_{S_{e}}\right)^{++}\right.$, $\mathrm{V}_{Z_{n}},\left(\mathrm{~V}_{Z_{n}}\right)^{--}, \mathrm{N}_{S_{e}}$, and $\left.\left(\mathrm{N}_{S_{e}}\right)^{-}\right]$, using a full-potential, linear combination of muffin-tin orbitals total energy calculation including an atomic force routine. We have obtained results for the lattice response of $\mathrm{ZnSe}$ in various configurations, and discuss these in comparison to the pseudopotential results and experiment. For the case of nitrogen substitution for selenium, we also present an independent experimental verification of previously reported results for the unusually large lattice relaxation surrounding this defect.
\end{abstract}

DOI: 10.1103/PhysRevB.64.035206

PACS number(s): 61.72.Bb, 61.72.Vv, 61.72.Ji

\section{INTRODUCTION}

The wide-band-gap semiconductor $\mathrm{ZnSe}$ and its alloys provide one of the most promising materials systems for blue-green-light-emitting diodes and lasers. Although feasibility has been demonstrated ${ }^{1}$ device degradation occurs in an unacceptably short time, with analysis indicating degradation is due to a high concentration of as-yet unidentified point defects. ${ }^{2}$ Considerable effort has focused on both theoretical and experimental methods for identifying the defects responsible. Several recent experimental studies ${ }^{3-7}$ indicate the presence of selenium vacancies as the most likely point defect to explain the observed data in molecular beam epitaxy-grown $\mathrm{ZnSe}$. However, theoretical treatments have led to conflicting views on the role of the selenium vacancy, or for that matter, of a number of other point defects. For example, the pseudopotential calculations of Laks et al. , $^{89}$ concluded that the formation energy of the various ionization states of the Se vacancy was too large to play a significant role in $\mathrm{ZnSe}$; whereas the pseudopotential calculations of Garcia and Northrup ${ }^{10}$ found that lattice relaxation could allow a doubly ionized Se vacancy as the most abundant native point defect in $p$-type $\mathrm{ZnSe}$. The importance of lattice relaxation has also been demonstrated in our preliminary reports. 11

From a theoretical point of view, ZnSe presents computational problems due to the existence of $\mathrm{Zn} 3 d$ semicore states. Although treating these states as core states significantly simplifies calculations, such a treatment results in a poor description of the bulk properties of zinc-based binary semiconductors. ${ }^{12}$ Consequently, in several more recent calculations $\mathrm{s}^{8,9,13,14}$ the $\mathrm{Zn} 3 d$ electrons were treated as valence electrons. However, the fact that these states are not well localized as core states, but still are not normal valence states, presents a challenge for the pseudopotential technique. Conventional pseudopotential calculations with a plane-wave basis set require too many waves, and that circumstance significantly reduces the computational benefits of pseudopotential calculations versus all-electron calculations. To overcome this complication, Laks et al. ${ }^{8,9}$ augmented the plane-wave basis by tight-binding orbitals. This approach allowed them significantly to improve the agreement between calculated and experimental values for the lattice constant and bulk modulus of $\mathrm{ZnSe}$. Consequently, they applied this approach for their study of defects, and we discuss this below.

Alternatively, in several publications ${ }^{10,15-19}$ the $\mathrm{Zn} 3 d$ electrons were left in the core shell, but a nonlinear corevalence (NCV) correction ${ }^{20}$ was used to account for the overlap between the $\mathrm{Zn} 3 d$ and the valence electrons. Use of the NCV correction provided a significant computational gain in comparison to calculations treating $3 d$ electrons as valence electrons, and therefore allowed consideration of larger defect systems. However, in the derivation ${ }^{20}$ of this correction a pseudopotential density rather than the real charge density was used inside the core region. (The pseudopotential approach allows electron density to deviate from the all-electron density in the core region.) Therefore, a careful comparison of these calculations utilizing the NCV correction with $a b$ initio all-electron full-potential calculations is desirable. Such a comparison is also needed to verify transferability of the pseudopotentials.

Although there are two such comparisons in the literature, ${ }^{13,17}$ they seem to provide contradictory results. Lee, Lee, and $\mathrm{Ihm}^{13}$ calculated several bulk properties, such as lattice constant, cohesive energy, and bulk modulus of $\mathrm{ZnSe}$ (and two other $\mathrm{Zn}$-based semiconductors) using all three methods listed above ( $3 d$ electrons in the core shell, in 
the core shells with the NCV correction, and $3 d$ electrons in the valence band) and made comparisons between the results obtained and corresponding experimental values. They concluded that the NCV correction provides only an insignificant improvement over the straightforward $3 d$-electron-in-core scheme. In contrast, their pseudopotential calculations with the $\mathrm{Zn} 3 d$ electrons in the valence band were in good agreement with experiment. On the other hand, Pöykkö, Puska, Korhonen, and Nieminen, ${ }^{17}$ in order to justify their pseudopotential approach based on the NCV correction, also performed all-electron full-potential (FP) calculations. They found good agreement between NCV-corrected pseudopotential and FP results not only for the bulk properties of pure $\mathrm{ZnSe}$, but also for two test cases of systems with defects-the doubly-positive Se vacancy and the doublypositive $\mathrm{Zn}$ interstitial. (References 13 and 17 do not provide sufficient details of the calculations to allow us to discuss the source of the disagreement between these two sets of results.) The existence of a difference between the formation energies of these two defects also means that the results of the $3 d$-in-valence calculations of Ref. 9 also deviated from all-electron calculations. However, we note that in their test all-electron FP calculations, Pöykkö et al. did not calculate lattice relaxation, but rather compared FP total energies of atomic configurations obtained by pseudopotential lattice relaxation calculations.

Thus, there are remaining methodological questions about calculations involving point-defect configurations in $\mathrm{ZnSe}$, let alone calculations involving defect complexes. To study these questions and independently test the core correction used in pseudopotential calculations we have employed an $a b$ initio, full-potential linear combination of muffin-tinorbitals (FP-LMTO) method ${ }^{21}$ including a force routine. ${ }^{22}$ This variant of the LMTO method provides results of high quality by including interstitial potentials and multiple energy windows. Thus, comparison of the results for point defect configurations obtained by this technique with the various results obtained in pseudopotential approaches can validate one of the prior calculations. Alternatively, such a comparison might indicate that additional critical thought must be given to the difference between pseudopotential (including those with NCV corrections) and full potential allelectron methods (such as LMTO), especially as to any underlying assumptions and approximations.

\section{METHODOLOGY}

Our approach allows a direct treatment of semicore electrons within a reasonably small number of basis states without the need for various corrections such as the pseudopotential calculations performed previously require. The details of this FP-LMTO method are described in Refs. 21 and 22. Here we summarize some of the features of the present method. Our FP-LMTO method of solving the bulk density functional problem includes a true interstitial region (with no atomic sphere approximation) and has a full potential, both in the muffin-tin and interstitial regions. In addition, the muffin-tin orbitals are not constrained to have zero kinetic energy in the interstitial. Use of both the full potential and the ability to optimize the parameters describing the basis states are essential in accurately calculating, within a LMTO approach, the total energies and electronic structure in lowsymmetry, open structures. Multiple kappas and energy windows provide basis enrichment that allows us the flexibility to capture the behavior of the zinc $3 d$ electrons. The kappas are energy parameters (in the case of valence electrons, they correspond to the square root of the average kinetic energy); so we have the ability to allow different types of electrons in a material to be characterized by their own kappas rather than being restricted to one kappa for all noncore electrons. By an "energy window," we mean a portion of the overall energy range over which the linearized electronic structure calculation is being done. There is a choice of basis functions to optimize the quality of calculation in the individual energy window. The multiple energy windows allow us to break the range of energy appropriate for each kappa into smaller, perhaps overlapping, more computationally manageable pieces. We also note that relativistic corrections are included in the calculations.

This self-consistent FP-LMTO method allows one to calculate the electronic band structure and corresponding charge density, total energy, and interatomic forces. It has been successfully applied to many systems. Both metallic and highly covalent materials have been studied. Lattice relaxation around defects and additives, surface and cleavage energies, and surface relaxations are a few examples of quantities that have been investigated.

To characterize $\mathrm{ZnSe}$ adequately required full use of the enhancements available in this method. The best results were obtained using four kappas (five for the case of nitrogen substitution) distributed in three separate energy windows. In choosing this way of organizing calculations we were initially guided by the results for systems"where the difference between the energy of the highest core states and the energy of the lowest valence states is relatively large (more than 2 Ry). In such systems the core states are well localized within the muffin-tin sphere and do not hybridize with valence states. In such cases it has proven sufficient to have two kappas for the valence electrons. Therefore we allowed two kappas for the valence states in $\mathrm{ZnSe}$. In addition, semicore zinc $3 d$ electrons were isolated by giving them their own kappa and energy window. Finally, it was found that the $4 \mathrm{~s}$ and $4 p$ electrons from the selenium atoms were participating in some covalent bonding with the zinc $3 d$ electrons; and so another kappa and energy window was allotted for them. With this choice of bases in the various energy windows, for $\mathrm{ZnSe}$ in the absence of defects we computed the lattice constant and found that it was less than $1 \%$ smaller than experiment, as expected for high-quality local density approximation calculations. We obtained a value of $64.9 \mathrm{GPa}$ for the bulk modulus that compares well with the typical values for various pseudopotential calculations and the experimental value of $62.5 \mathrm{GPa}$. Table I lists our results along with experiment and previously calculated numbers for the lattice constant and bulk modulus. Also, the band structure and density of states were obtained and gave excellent agreement with previous results. ${ }^{23}$

To perform the defect calculations we used a supercell 
TABLE I. Lattice constant and bulk modulus for $\mathrm{ZnSe}$.

\begin{tabular}{lccc}
\hline \hline & Experiment & Present calculations & Other calculations \\
\hline Lattice constant $(\AA)$ & 5.669 & 5.636 & $5.680,{ }^{\mathrm{a}} 5.677,{ }^{\mathrm{b}} 4.916,{ }^{\mathrm{c}} 5.198,{ }^{\mathrm{d}} 5.592,{ }^{\mathrm{e}} 5.638,{ }^{\mathrm{f}} 5.61, \mathrm{~g}$ \\
Bulk modulus (GPa) & 62.5 & 64.9 & $63.2,{ }^{\mathrm{a}} 68.9,{ }^{\mathrm{b}} 131.7,{ }^{\mathrm{c}} 98.9,{ }^{\mathrm{d}} 70.1,{ }^{\mathrm{e}} 65.2,{ }^{\mathrm{f}} 81, \mathrm{~g}^{\mathrm{g}}$ \\
\hline
\end{tabular}

${ }^{\mathrm{a}}$ Reference 15 with partial core correction for $\mathrm{Zn}$ potentials.

beference 15 with $3 d$ electrons treated as valence electrons.

${ }^{c}$ Reference $13 \mathrm{Zn}$-3d-in-core.

${ }^{d}$ Reference 13 with NCV corrections.

'Reference $13 \mathrm{Zn}-3 d$-in-valence.

'Reference $13 \mathrm{Zn}$-3d-in-valence and Se-3d-in-valence.

Reference 17.

methodology. In this approach, the defect is surrounded by the host atoms in a simulation cell that is periodically repeated to fill all space. There are two problems associated with this approach: (1) the artifact of having an interaction between defects belonging to adjacent simulation cells, and (2) the presence of symmetry constraints that restrict lattice relaxation around the defects. To assess and deal with these problems, we have found the relaxed lattice structure for vacancies in both 16- and 32-atom unit cells and compared the results.

We start from the ideal lattice for $\mathrm{ZnSe}$ and remove one atom from either the zinc or selenium location to consider vacancies, or replace one selenium atom with nitrogen. Then we self-consistently solve, using our LMTO code, for the full potential and total energy. Once convergence is obtained, the force routine is applied, which gives us the interatomic forces on each atom in the cell. The result is a force vector whose components correspond to the available degrees of freedom allowed by the particular system being studied. The atoms are then moved in the direction given by the force vector. A distance along that vector is determined by a conjugate gradient scheme using previous positions and forces. Then the procedure is repeated, self-consistently solving for the total energy for this new configuration, obtaining the force vector and moving the atoms. The process is repeated until the total energy stabilizes at its lowest value.

When we considered charged defects, a neutralizing uniform background charge was added to avoid Coulomb interaction between supercells. We will show that a 16-atom unit cell is not sufficient to describe point defects in $\mathrm{ZnSe}$ even for a charge neutral case. Therefore we consider charged defects only with a 32-atom unit cell.

\section{RESULTS AND DISCUSSION: Se AND Zn VACANCIES, N SUBSTITUTED FOR Se}

We have found the relaxed lattice structure for vacancies and nitrogen substitution for selenium in both 16- and 32atom unit cells. We first consider the case of a Se vacancy to illustrate the details of 16- and 32-atom cells in terms of symmetry and degrees of freedom, and why the larger supercell is necessary. The same symmetry considerations apply for the $\mathrm{Zn}$ vacancy and nitrogen substitution described later in the text.

\section{A. Selenium vacancy}

The conventional 8-atom $\mathrm{ZnSe}$ unit cell is shown in Fig. 1. There we see the zinc blende structure with the selenium atoms occupying the eight corners and face centers of the six faces of the cell forming a face-centered-cubic (fcc) lattice. [From the computational point of view, it is convenient to have the origin coincide with the site marked "1" in Fig. 1. In this case removing the atom and creating a vacancy on this site does not change the overall symmetry of the simulation cell. (Below we will use the terms "supercell" and "simulation cell" interchangeably).] The simplest way to begin our discussion is to consider the conventional supercell shown in Fig. 1. Such a 7-atom-plus-a-single-vacancy supercell has a convenient cubic symmetry. All corner atoms shown in Fig. 1 belong to neighboring supercells, except " 1 ," which is chosen as the vacancy site. Therefore, when this eight-atom supercell is repeated periodically to fill all space, all corner atoms in Fig. 1 (i.e., sites numbered 5, 6, and 7 as well as 1) are substituted by vacancies, totally changing the initial zinc blende structure. To go to a larger size supercell, we considered 16- and 32-atom supercells, which can be generated by including one or three cells adjacent to the one containing the four $\mathrm{Se}$ and four $\mathrm{Zn}$ atoms as shown in Fig. 1.

Removing one of the selenium atoms in the 16-atom unit cell results in four corner atoms disappearing from the original 8-atom cell. In Fig. 1 these are atom " 1 " and the three atoms marked as " 5 ." It can be seen that this arrangement has the vacancies form a fcc lattice with basis vectors twice as long as those for the original fcc Se sublattice. When repeated periodically to fill all space, the remaining corner Se atoms (atoms 6 and 7 in Fig. 1) are surrounded equidistantly by the vacancies in 12 different supercells, and thus are constrained to be fixed. Examining the zinc atoms in this configuration, it can be seen that all four zinc atoms in Fig. 1 (atoms marked 2 and 4) are adjacent to vacancy sites and, consequently, are equivalent by symmetry. The other four zinc atoms for the 16-atom supercell belong to one of the neighboring conventional 8-atom cells (e.g., the one on top of the cube shown in Fig. 1), and these atoms are not adjacent to the vacancy.

When describing the relaxation motion of the atoms belonging to the two nearest shells, it is more convenient to consider a different projection for the figures. In Figs. 2, 3, 


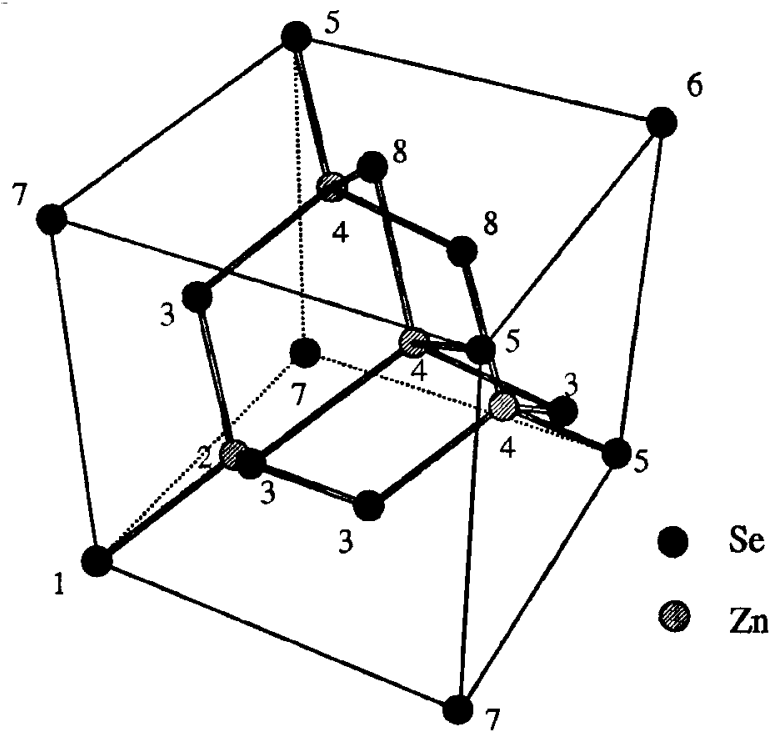

FIG. 1. The conventional eight-atom unit cell (four atoms inside the cube, plus one-eighth of each of eight corner atoms, plus onehalf of each of six face-center atoms) of the $\mathrm{ZnSe}$ zinc-blende lattice with $\mathrm{Se}(\mathrm{Zn}$, when $\mathrm{Zn}$ vacancy is discussed) atoms represented by solid circles and $\mathrm{Zn}(\mathrm{Se})$ atoms by crosshatched circles. The Se at the origin is labeled 1 and the three Se's at face centers are labeled 3. The corresponding $\mathrm{Zn}$ atoms are labeled 2 and 4 . Bonds between nearest neighbors, as well as cube edges, are shown to guide the eye. The numbering of atoms is done for convenience in describing the 16- and 32-atom supercells. Atoms of type 5, 6, and 7 are the origins of the adjacent conventional cells. The vacancy is created by removing an atom from site 1 . For the 16-atom supercell, vacancies would also occupy sites 5 , and for the 32-atom supercell, site 6 . Atoms of type 2 are always next to the vacancy, while the atom of type 4 has a next-neighbor vacancy in the 16-atom supercell and a next neighbor $\mathrm{Se}(\mathrm{Zn})$ atom in the 32-atom supercell. To form a 16-atom supercell we need to include atoms from an adjacent cube (e.g., the cube on top of the one shown in this figure), while for a 32-atom supercell, atoms from an additional three adjacent cubes are needed. In practice, in order to reduce computing, these atoms are rearranged to bring them closer to the origin and increase the symmetry.

and 4 we show the vacancy site in the center, surrounded by two atomic shells. For the second shell we show only nine out of the 12 selenium atoms, with three selenium atoms removed in order to provide a better view. All four zinc atoms from the first nearest-neighbor shell can only move directly toward or away from the nearest vacancy as depicted in Fig. 2. The face-centered selenium atoms (i.e., atoms " 3 " in Fig. 1) form the second neighbor shell. The motion of atoms in this shell is shown in Fig. 3. This motion is restricted by their neighboring $\mathrm{Zn}$ atoms (j.e., atoms marked " 2 "' and " 4 " in Fig. 1) being identical by symmetry. Hence selenium atoms " 3 " can move only perpendicular to the plane of cubic faces (see Fig. 1) and, therefore, cannot follow zinc atoms from the first shell when they move toward (or away) from the vacancy.

Further investigation reveals that there are only three degrees of freedom for relaxation in the 16-atom supercell. The remaining, third, degree of freedom allows zinc atoms in the

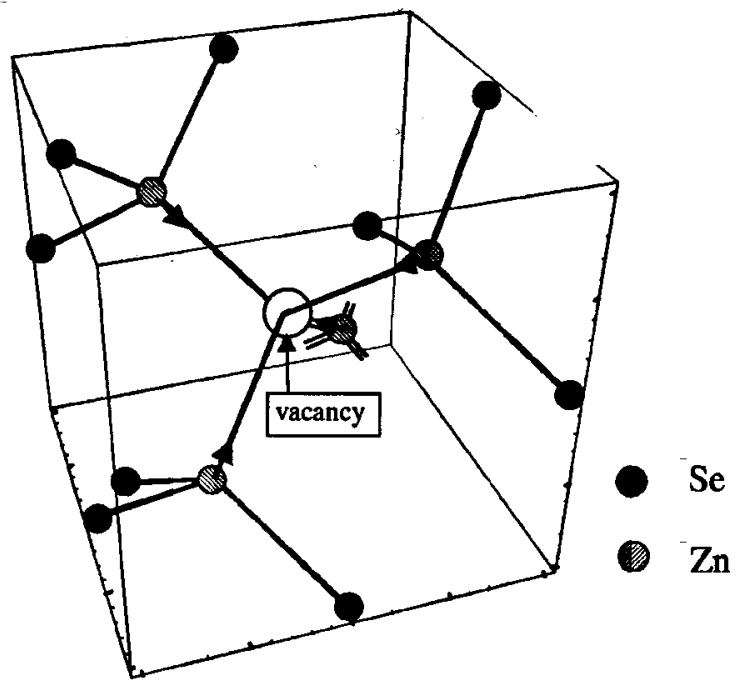

FIG. 2. Symmetry-allowed relaxation around vacancy for the first atomic shell (for both 16-atom and 32-atom supercells). Four $\mathrm{Zn}$ atoms (or Se for the $\mathrm{Zn}$ vacancy), which are shown as crosshatched circles are allowed to move directly toward or away (as shown by the arrows) from the vacancy site that is located in the center. Symmetry demands equal distances between the origin (which coincides with the vacancy site) and each of these atoms. Nine of the 12 atoms from the second atomic shell are shown as filled circles. These are Se atoms in the case of a Se vacancy and $\mathrm{Zn}$ atoms in the case of a $\mathrm{Zn}$ vacancy. Three atoms from the second shell are removed from the figure to facilitate viewing. Atoms of the second shell are atoms of type 3 from Fig. 1, and are located at the centers of the cubic faces of the conventional fcc cube.

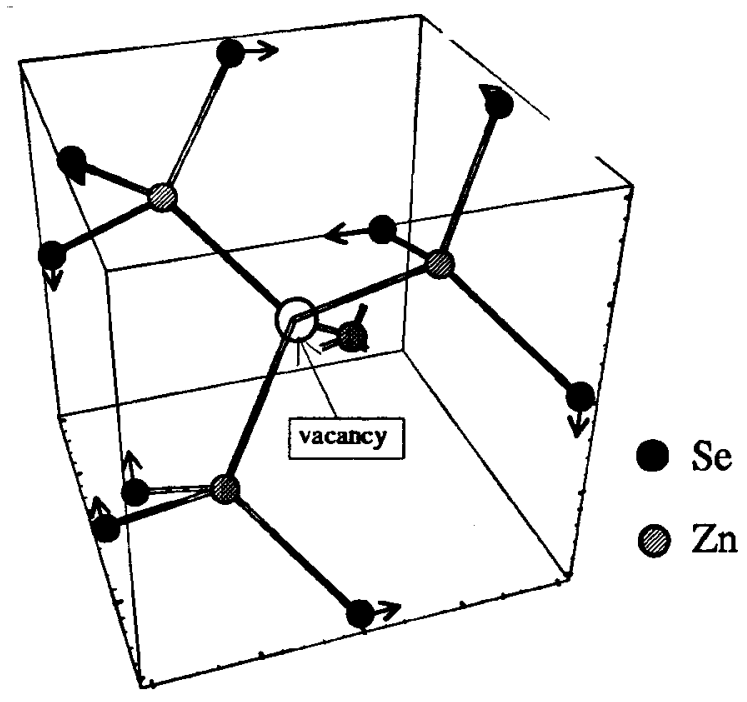

FIG. 3. Relaxation of atoms around a Se vacancy in a 16-atom supercell. Atoms shown in this figure are the same as in Fig. 2. Arrows demonstrate the only available degree of freedom for relaxation motion of the second atomic shell. This motion is in the direction perpendicular to the planes of the cubic faces in Fig. 1 (atoms of type 3). Therefore these atoms cannot follow atoms from the first shell in their motion. 


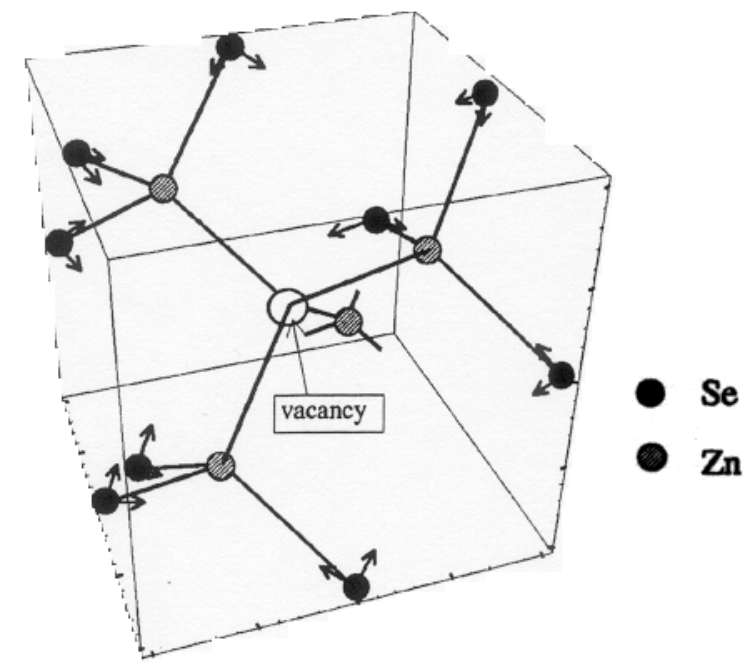

FIG. 4. The same as Fig. 3 for 32-atom supercell. Now, both "tangential" and "radial" motions are available for the atoms of the second shell. Thus a greater relaxation motion is expected than for 16-atom supercell.

8-atom cube just above the one shown in Fig. 1 (i.e., making up the remainder of the 16-atom supercell) to move directly toward or away from their nearest fixed selenium neighbor. This artificially separates the zinc atoms of the third atomic shell into two groups, namely atoms like those marked " 4 " in the cube shown in Fig. 1 that are adjacent to a vacancy, versus zinc atoms from the adjacent cube, such as nearest neighbors of the atom marked " 7 "' in Fig. 1. This built-in artifact of the symmetry of this 16-atom supercell can thus be seen to partition the zinc atoms into clusters interior to these cubes. These clusters prevent the forming of a shelllike arrangement that can more realistically accommodate the lattice relaxation around a defect.

We can compare this to the 32-atom unit cell. Now, when a Se vacancy is created, only two of the eight corner $\mathrm{Se}$ atoms specifying the 8-atom cell need be removed. In Fig. 1 these atoms are labeled as 1 and 6 . This generates a bodycentered-cubic (bcc) lattice of vacancies with a supercell that effectively includes four of the conventional zinc-blende cubes, although in practice these atoms are rearranged to place them as close to the origin (which is the site of the vacancy) as possible. This arrangement results in atoms of four distinct symmetry types (plus the vacancy) with five degrees of freedom to move. It is easy to see that now there are only two types of zinc atoms, namely, those with a vacancy for a nearest neighbor (i.e., atoms of type 2) and those without (i.e., of type 4). Again, all remaining corner Se atoms (atoms of type 5 and 7) are constrained by symmetry to remain fixed, but now the face-centered Se atoms have two degrees of freedom as is shown in Fig. 4. Selenium atoms now can follow the motion of zinc atoms from the first shell, therefore facilitating their relaxation motion. The zinc atoms nearest to a vacancy are still constrained to move only directly toward or away from that vacancy (Fig. 2), but the other zinc atoms now have two degrees of freedom; one being again radially toward or away from its nearest fixed selenium neighbor, while the other is an angular component allowing the bond to rotate in the (111) plane. In this supercell, there is a more physically appropriate division of the atoms into symmetrically equivalent types that form a roughly shell-like structure around the vacancy, as opposed to the highly artificial cluster structure imposed by the 16atom supercell symmetry described above. Hence, it is reasonable to expect that relaxation can be captured more realistically in this 32-atom supercell.

We now compare the relaxation around vacancies found in each of these supercells. As a characteristic length it is convenient to use the equilibrium length of the shortest bond, i.e., the nearest-neighbor distance (NND). A summary of calculated results is shown in Table II.

It can be seen that in the 16-atom supercell there is a significant relaxation displacement of zinc atoms nearest the neutral selenium vacancy (around 5\% of NND). Motion of selenium atoms from the second shell is negligible. In the 32-atom supercell, atoms from the first shell move even further (14.8\% of NND), while displacement of the second shell atoms from their initial positions is equal to $3.4 \%$ of NND. This displacement is mostly ( $3.2 \%$ of NND) directed toward the vacancy, taking advantage of the available "radial" degree of freedom (see Fig. 4 in comparison to Fig. 3). Although this relaxation motion is relatively small, there are 12 atoms of this kind versus only four atoms from the first shell. Therefore the cumulative effect of this motion represents a

TABLE II. Relaxation around defect site. In this table we used the following notations. $N$ is a number of atoms in the supercell. $E_{\text {relax }}$ is a relaxation energy. $\mathrm{RD}_{\text {first }}$ is a relative change in the distance from the vacancy for the first neighboring shell (atom 2 in Fig. 1) as a percentage of NND. RD second and TD $_{\text {second }}$ are correspondingly radial and tangential displacements of atoms in the second neighboring shell (atom 3 in Fig. 1) as a percentage of NND.

\begin{tabular}{lccccccccc}
\hline \hline & & $\mathrm{V}_{\text {Se }}$ & & $\left(\mathrm{V}_{\text {Se }}\right)^{++}$ & \multicolumn{2}{c}{$\mathrm{V}_{\mathrm{Zn}_{n}}$} & $\left(\mathrm{~V}_{\mathrm{Zn}_{n}}\right)^{--}$ & $\mathrm{N}_{\text {Se }}$ & $\left(\mathrm{N}_{\text {Se }}\right)^{-}$ \\
\hline$N$ & 16 & 32 & 32 & 16 & 32 & 16 & 32 & 32 \\
$E_{\text {relax }}(\mathrm{eV})$ & -0.08 & -0.44 & -1.2 & -0.13 & -0.21 & -2.1 & -2.7 & -2.8 \\
$\mathrm{RD}_{\text {first }}$ & $-4.97 \%$ & $-14.8 \%$ & $+19.4 \%$ & $-4.74 \%$ & $-7.2 \%$ & $-9.7 \%$ & $-20.0 \%$ & $-19.0 \%$ \\
$\mathrm{RD}_{\text {second }}$ & $0^{\mathrm{a}}$ & $-3.2 \%$ & $0.4 \%$ & $0^{\mathrm{a}}$ & $-2.6 \%$ & 0 & $-3.5 \%$ & $2.0 \%$ \\
$\mathrm{TD}_{\text {second }}$ & $0.4 \%$ & $1.0 \%$ & $2.6 \%$ & $3.4 \%$ & $2.3 \%$ & $0.5 \%$ & $4.9 \%$ & $0.2 \%$ \\
\hline
\end{tabular}

${ }^{\text {a }}$ Radial relaxation is not allowed by the symmetry. 
significant relaxation in going to the final equilibrium configuration.

Other results of our calculations for a neutral Se vacancy are as follows. In the 16-atom supercell, the decrease in the distance from the vacancy to nearest-neighbor $\mathrm{Zn}$ atoms forces the remaining $\mathrm{Zn}-\mathrm{Se}$ bonds to become longer. The bonds between $\mathrm{Zn}$ (atoms 2 and 4 in Fig. 1) and corner Se atoms (atoms 7 in Fig. 1) increase by $0.5 \%$, and the bonds between $\mathrm{Zn}$ atoms and face-centered Se atoms (atoms 3 and 8 in Fig. 1) atoms increase by $1.5 \%$. In the 32-atom supercell most of the motion is also limited to the first and second shells of atoms. The remaining $\mathrm{Zn}$ atoms move slightly, ending up $1.35 \%$ further from their fixed Se neighbor. The bonds between the nearest-neighbor zinc atoms and their face-centered selenium neighbors (atoms 2 and 3 on Fig. 1) increase in length by $2.8 \%$.

Because of geometrical differences and additional degrees of freedom in the 32-atom cells as compared to the 16-atom cells, as described above, the relaxation energy for the 32atom cell with selenium vacancy is more than five times higher than for the 16-atom unit cell $(-0.44$ versus $-0.08 \mathrm{eV}$ ). We also used the 32-atom supercell to calculate relaxation around a doubly positively charged Se vacancy. There is much stronger lattice relaxation for the charged vacancy than for the neutral vacancy. The relaxation energy is $-1.2 \mathrm{eV}$, and the neighboring zinc atoms move away from the vacancy by $19.4 \%$ of NND. Thus the direction of the relaxation of the neighboring atoms reverses its sign for charged versus neutral vacancies.

Garcia and Northrup ${ }^{10}$ reported relaxation energies for the same two types of Se vacancies and obtained $-0.15 \mathrm{eV}$ for the neutral and $-1.61 \mathrm{eV}$ for the doubly ionized vacancy; while Pöykkö and co-workers found ${ }^{18}$ relaxation energies of $-0.49 \mathrm{eV}$ and $-1.58 \mathrm{eV}(-1.4 \mathrm{eV}$ in Ref. 17) for these two cases, respectively. Our results for the neutral case are much closer to the results of Refs. 17 and 18. For the charged case our relaxation energy $(-1.2 \mathrm{eV})$ is smaller than in both pseudopotential calculations, but agrees well with the $-1.1 \mathrm{eV}$ obtained by the FP-LMTO calculations of Ref. 17. (The $0.1 \mathrm{eV}$ difference in FP-LMTO calculations can be understood from the fact that lattice relaxation in Ref. 17 was not calculated in FP-LMTO, but rather the final atomic configuration obtained in pseudopotential calculations was used. We also note that the FP-LMTO method used in Ref. 17 was not the same as ours, but rather was that of Methfessel ${ }^{24}$ that has methodological difference from ours.) Finally, our predicted $19.4 \%$ outward movement for the nearest-neighbor zinc atoms for the doubly ionized vacancy agrees well with the $20 \%$ of Ref. 10 and $24 \%$ of Ref. 18. Therefore our overall results are in good quantitative agreement with results obtained by Pöykkö and co-workers. ${ }^{17,18}$

Some important information can be obtained by studying changes in the charge distribution in the region surrounding defects, especially changes inside the muffin-tin spheres of the neighboring atoms. In particular, this can be used to check the adequacy of the size of the defect-containing supercell. If the charges on the outermost (from the point defect) atoms in the supercell deviate significantly from the charges in the ideal, defect-free lattice, the size of the supercell is too small. For such a supercell, an artificial interaction between defects in the adjacent supercells cannot be neglected; and a larger supercell has to be used in order to describe a single isolated defect.

In FP-LMTO calculations the charges inside the muffintin sphere depend on the radii of these spheres. Therefore, it is worthwhile to mention here a strategy that we adopt for choosing these radii. The first point in this strategy is that radii of mufin-tin spreres should remain constant, while overlapping between muffin-tin spheres should be avoided during the entire course of relaxation calculations, i.e., on going from an initial, undistorted lattice to a completely relaxed lattice that corresponds to the minimum of the total energy for a given simulation cell. On the other hand, it is beneficial to have as much volume as possible inside muffin tins, since a reduction of the interstitial volume decreases the size of the calculations (because of the smaller number of Fourier harmonics required) and increases the accuracy of the calculations. To accommodate these conflicting demands, we adopt an empirical rule that the minimum gap between any two muffin-tin spheres should be between $5 \%$ and $15 \%$ of the distance between their centers. A touching of muffin-tin spheres is avoided in order to prevent large gradients of the potential in these areas. Simultaneously, we maintain the same muffin-tin radius for the same species of atoms in order to control the accuracy of supercell calculations. (For an ideal lattice, charges inside muffin-tin spheres should be identical for all atoms of the same species). As a result of these considerations, for the present calculations, radii for $\mathbf{Z n}$ and Se muffin-tin spheres were chosen to be 2.2 atomic units for both types of atoms.

In the case of the neutral selenium vacancy in the final, relaxed lattice structure, the four zinc atoms surrounding a vacancy lose 0.12 electrons each. Each of 12 selenium atoms from the second shell lose 0.02 electrons to the interstitial area. It is interesting that in the unrelaxed lattice, atoms of the second shell around the vacancy do not lose any charge when compared to the selenium atoms in the initial, vacancyfree ideal lattice (total loss is less than 0.01 electron for 12 atoms).

For the doubly positive Se vacancy each of the four nearest zinc atoms loses 0.03 electrons, while selenium atoms from the second shell lose 0.023 electrons each. This number goes down beyond the second shell to 0.009 for the third and 0.008 for the fourth. This relatively fast charge relaxation indicates that the 32-atom cell is probably sufficient for these calculations.

\section{B. Zinc vacancy}

The same symmetry considerations as we used for the selenium vacancy apply to the relaxation about a zinc vacancy. However, when compared to the selenium vacancy calculations, the movement of the selenium atoms nearest the vacancy is smaller for the 32-atom cell (see Table II). In the 16-atom supercell the nearest-neighbor $\mathrm{Se}$ atoms to the vacancy decrease their distance from the vacancy by $4.7 \%$. 
This forces the bonds between fixed corner $\mathrm{Zn}$ atoms and Se atoms (e.g., bonds between atoms 7 and 2) to become $0.5 \%$ longer, and bonds between the movable face-centered $\mathrm{Zn}$ atoms and Se atoms (e.g. atoms 3 and 2) to shrink by $0.2 \%$. In the 32-atom supercell, the nearest-neighbor $\mathrm{Se}$ atoms are now $7.2 \%$ closer to the vacancy. The remaining Se atoms move little, ending up only $0.5 \%$ further from their fixed $\mathrm{Zn}$ neighbor. The bonds between the nearest-neighbor Se atoms and their face-centered zinc neighbors decrease in length by $0.9 \%$. The other selenium bonds for the face-centered zinc atoms increase by $1.1 \%$. The relaxation energies per vacancy are $-0.13 \mathrm{eV}$ for the 16 -atom supercell and $-0.21 \mathrm{eV}$ for the 32-atom supercell. Thus the difference between 16- and 32-atom cells is much smaller than for the Se vacancy as reported above.

For the doubly negative zinc vacancy in the 32-atom supercell the relaxation of the nearest neighbors is similar to the neutral case, unlike the behavior for the selenium charged vacancy. Neighboring selenium atoms move toward the vacancy by $6.6 \%$ of the NND, and the relaxation energy is $-0.38 \mathrm{eV}$. This is again reasonably close to the results reported by Ref. 18, $-0.54 \mathrm{eV}$.

The selenium atoms neighboring the neutral zinc vacancy lose about 0.08 electrons each. The charge redistribution in the second shell from the vacancy follows a similar pattern to the case of the selenium vacancy. The 12 zinc atoms from the second shell do not change their charge prior to relaxation, losing only 0.012 electrons in total, while after relaxation this number grows to 0.168 electrons. This serves as an indication that the direct influence of the vacancy is limited only to the atoms of the first shell. The charge redistribution at the atoms of the second and following shells (for which the charge changes very little) is not caused directly by the vacancy itself, but rather by the lattice relaxation, and by the consequent change in distances to neighboring atoms.

For the doubly negative zinc vacancy, there is no noticeable change in the charge inside muffin-tin spheres for all atoms in the supercell when compared to the corresponding values of the ideal lattice. The change in charge is 0.008 electrons loss per sphere in the first shell and a gain of about 0.01 electrons per sphere in other shells.

\section{Low-symmetry supercell for zinc vacancy}

Note that the high-symmetry configuration for the $\mathrm{Zn}$ or Se vacancy systems, as described above, constrains all four nearest-neighbor $\mathrm{Se}$ atoms (or $\mathrm{Zn}$ atoms in the case of the $\mathrm{Se}$ vacancy) to be the same distance from the $\mathrm{Zn}$ vacancy. Since our calculation takes advantage of the symmetry to reduce the amount of computation needed, the forces are constrained to be in only those directions that will not lower the symmetry of the supercell, as illustrated in Fig. 2. Therefore, to reach any lower symmetry as a final relaxed state, this symmetry must be broken "by hand" in the original construction of the supercell. We were experimentally motivated $^{25}$ to release this constraint while considering a zinc vacancy. Therefore we considered a lower-symmetry (trigonal) configuration in which only three of the four nearest-neighbors were at the same distance from the va-

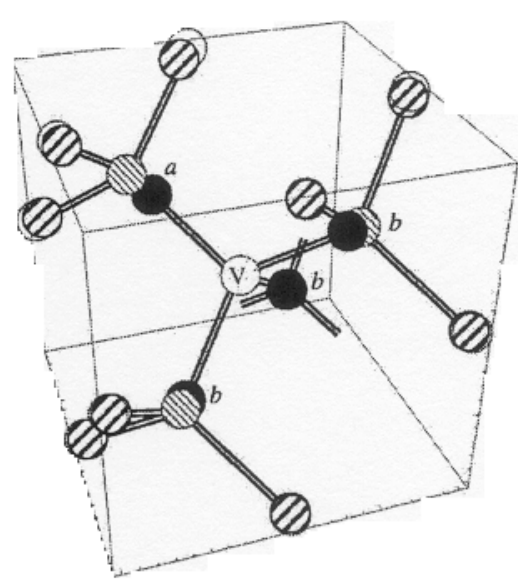

Zn aroms

FIG. 5. Relaxation of atoms around a $\mathrm{Zn}$ vacancy in the lowsymmetry 32-atom supercell. Calculated positions of atoms are shown in this figure. In the lower symmetry (trigonal) configuration only three of the four nearest neighbors (type $b$ ) are required to be at the same distance from the vacancy, while the remaining Se nearest-neighbor atom (type $a$ ) is free to move independently. The asymmetric nearest-neighbor atom is $12.4 \%$ closer to the vacancy, while the remaining three nearest-neighbor atoms are $7.7 \%$ closer to the original position of the vacancy. These three atoms no longer move only toward the vacancy; reduced symmetry allows them to relax away from each other by changing their angle with respect to each other and the single nearest-neighbor atom. Zinc atoms of the second shell follow atoms of type $b$ in their relaxation motion toward the vacancy. The additional flexibility of low-symmetry relaxation results in much greater relaxation energy than in the case of high symmetry, more than $-0.36 \mathrm{eV}$ compared to about $-0.21 \mathrm{eV}$ for the high-symmetry 32-atom supercell.

cancy, while the remaining Se nearest-neighbor atom was free to move independently. As shown in Fig. 5 this resulted in very much greater relaxation for the asymmetric atom. The asymmetric nearest-neighbor atom (marked " $a$ " in Fig. 5 ) is $12.4 \%$ closer to the vacancy, while the remaining three nearest neighbor atoms (marked " $b$ " in Fig. 5) end up 7.7\% closer to the vacancy. These three atoms no longer move only toward the vacancy as in the symmetric supercell described above. Reduced symmetry allows them to relax away from each other by changing their bond angle with respect to each other and the single nearest-neighbor atom. Most of the remaining bond lengths change slightly as in the higher symmetry case, but now we have a great deal of change in the direction of the bonds relative to the original configuration. The 32-atom unit cell contains nine (plus vacancy) different types of atoms with 19 different degrees of freedom to move. This results in much greater relaxation energy than previously, more than $-0.36 \mathrm{eV}$ compared to about $-0.21 \mathrm{eV}$. These results indicate that the low-symmetry configuration around the zinc vacancy is highly preferable. However, calculations using low-symmetry supercells are extremely computer extensive in comparison to calculations using highsymmetry supercells. For the high-symmetry 32-atom cell (see Fig. 4), there are only five types of atoms with a total of five degrees of freedom, i.e., some with no degree to move and some with more than one degree of freedom. For this 


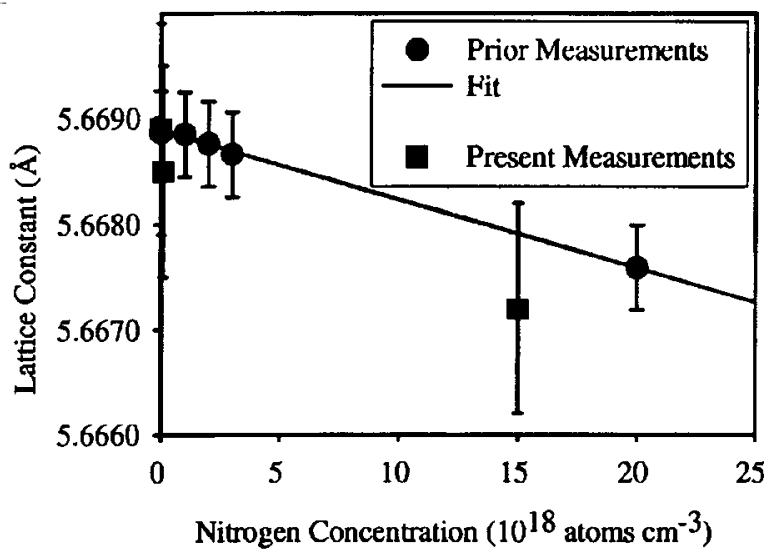

FIG. 6. Plot of lattice constant $(\AA)$ vs nitrogen concentration substituted for Se in the $\mathrm{ZnSe}$ crystal from prior work (Ref. 26) and our present measurements. The solid line is a fit to the data from Ref. 26.

reason we performed low-symmetry calculations only for the case of the neutral $\mathrm{Zn}$ vacancy.

\section{Nitrogen substitution for selenium}

Both experimental results by Petruzzello et al. ${ }^{26}$ and our present measurements show that incorporated nitrogen dramatically changes the structure of the local $\mathrm{ZnSe}$ lattice. The experimental dependence of the lattice constant on the nitrogen concentration is shown in Fig. 6. Both, the measurements in Ref. 26 and our measurements relied on conventional $x$-ray diffraction determination of the lattice constant using multiple diffraction spots to eliminate strain effects, with the nitrogen concentration determined using secondary mass spectrometry. The measured values and trends of the present and prior measurements agree within experimental uncertainty.

A simple experimental estimate of the equilibrium bond length between $\mathrm{Zn}$ atoms and $\mathrm{N}$ substitutional atoms can be obtained by assuming a linear change in the average lattice constant as measured by $\mathrm{x}$-ray diffraction with increasing molar fraction of substitutional nitrogen. Such use of this so-called "Vegard's law" approximation results in the linear fit shown in Fig. 6, and indicates an effective lattice constant of $4.24 \AA$ for zinc-blende $\mathrm{ZnN}$. This is $75 \%$ of the experimental lattice constant for $\mathrm{ZnSe}$ (see Table I). This suggests that the zinc atoms nearest to the diluted substitutional nitrogen would be more inward by about $25 \%$ of the initial $\mathrm{Zn}-\mathrm{Se}$ distance. Our FP-LMTO calculations for zinc-blende $\mathrm{ZnN}$, which does not exist experimentally, give a lattice constant of $4.5 \AA$. Agreement with the experimental extrapolation is excellent, considering that measurements exist only for very low concentrations of nitrogen (about $0.1 \%$ molar concentration) and that we have extrapolated to $50 \%$ concentration.

For the substitution of a single selenium atom by a nitrogen in $\mathrm{ZnSe}$ we have obtained results for the high-symmetry 16-atom and high-symmetry 32-atom unit cells, i.e., the same cells as used in the Se vacancy calculations. Our results show a stronger relaxation around the nitrogen atom than in case of the selenium vacancy. As shown in Table II, for the 32-atom cell, the relaxation energy is $-2.7 \mathrm{eV}$, while the nearest zinc bonds become $20 \%$ shorter, and the first-shell zinc-selenium bonds shrink by $4 \%$. For a negatively charged nitrogen substitution, results are very similar to the neutral case, with a relaxation energy of $-2.8 \mathrm{eV}$ and the nearest zinc bonds becoming $19 \%$ shorter.

These results show somewhat greater relaxation than pre-viously calculated results obtained using pseudopotential methods, ${ }^{14,15,18}$ which found $14-18 \%$ nearest-neighbor relaxation compared with our result of $19-20 \%$ and the result from extrapolation of experiment $25 \% .^{26}$ Alternatively we can compare results of the lattice relaxation around the nitrogen impurity with the zinc-nitrogen bond length in $\mathrm{Zn}_{3} \mathrm{~N}_{2}$ compound that exists in a cubic Bixbyte structure. ${ }^{27}$ This bond length is 2.08 that is $15 \%$ shorter than calculated 2.44 zinc-selenium bond length in zinc-blende lattice structure at our calculated lattice constant (see Table I). Thus, our predicted lattice relaxation is in very good agreement with experiment since it is exactly halfway between the two experimentally defined expectations ( $25 \%$ and $15 \%$ ). We note that the pseudopotential results fall more toward the lower of the experimentally indicated limits.

Analysis of the charge redistribution shows that in the case of neutral nitrogen substitution for selenium even the 32-atom unit cell size might not be sufficient. Since even in that case, for atoms further away from the nitrogen, the charge was not restored to its value in the ideal lattice. Nitrogen attracts too much negative charge, and selenium atoms of the second and the fourth shell ended up losing about 0.035 electrons each, and zinc atoms of the first and third shells lose 0.01 and 0.02 electrons each. However, for the negatively charged nitrogen substitution for selenium, changes in charge are localized to the first (loss of $0.02 \mathrm{elec}-$ trons per atom) and to the third (gain of 0.02 electrons per atom) shells; while changes in the charge of other atoms in the supercell are less than 0.004 electrons per atom.

\section{CONCLUSIONS}

The LMTO method is an all-electron method that has allowed us to execute a straightforward treatment of states with small negative energy ("semicore" states) as valence states. The full charge of the core states enters into the calculated charge density and so may influence such quantities as the equilibrium lattice constant through affecting the valence electrons. However, the core states are calculated in a spherically symmetric potential and are not allowed to hybridize with neighboring core states or valence orbitals. In cases where some core states substantially leak outside of the muffin-tin spheres and overlap with valence electrons, it is essential for accurate results to move the core states in question, i.e., "semicore" states, into the valence set of states. This allows accurate calculation of the physical effects of the "semicore" states within the full-potential methodology and enables their participation in hybridization. In practice this is accomplished through the flexibility available in enriching the basis provided by having additional energy windows. We found that for $\mathrm{ZnSe}$, the zinc $3 d$ and selenium $4 s$ and $4 p$ 
states do participate in some covalent bonding and hybridization. Pseudopotential calculations for $\mathrm{Zn}$ or $\mathrm{Cd}$ materials, which do not include $\mathrm{Zn}$ or $\mathrm{Cd} d$ levels in any way, obtain substantial errors. ${ }^{12}$ Those pseudopotential calculations ${ }^{8,9}$ that provide complete inclusion of the semicore levels should be expected to have the same levels of accuracy and reliability as those of a full-potential, all-electron calculation. However, as noted, we do find differences. Some of the calculations based on nonlinear core-valence correction in the form developed in Ref. 20 provide much better results. We found good quantitative agreement with Ref. 18. However, there are significant differences with results of another group ${ }^{13}$ using the same corrections. (References 13 and 18 do not provide sufficient details of the calculations to allow us to recognize the origin of the differences in their results.)

In contrast to the pseudopotential calculations of Ref. 18, in our LMTO calculation we include the selenium $4 s$ states as well as the zinc $3 d$ states in the valence set of states. In addition there is a not-very-well-studied question of transferability of pseudopotentials to calculations of non-neutral defects. These points, in conjunction with other differences in treating core and semicore electrons, may explain the remaining difference between our results and the pseudopotential calculations of Ref. 18 that do correct for semicore levels. Additionally, this difference may be related to the way in which nonlinear core corrections account for the large overlap of the zinc $3 d$ semicore electrons with the other components of the valence charge density; whereas our fullpotential LMTO methodology treats these electrons in the same manner as it treats valence electrons in other energy windows.

In conclusion, we emphasize that 16-atom supercells are not large enough to capture the relaxation around a point defect for $\mathrm{ZnSe}$, but 32-atom results give evidence of being quite reliable. This is because of the shell-like division of atoms in the 32-atom supercell, and the almost negligible movement of those atoms forming the shell furthest from the defect. It seems clear that atoms adjacent to the defect must be separated by more than one common atom in order to allow sufficient freedom of motion to adequately capture the lattice relaxation. We also have shown in the case of the $\mathrm{Zn}$ vacancy that better agreement with experiment, as compared to the high-symmetry supercell calculations, may be obtained by allowing the possibility of lowering the symmetry around the defect.

\section{ACKNOWLEDGMENT}

This research was supported by National Science Foundation Grant No. DMR-98-06299.
${ }^{1}$ S. Taniguchi, T. Hino, S. Itoh, K. Nakano, N. Nakayama, A. Ishibashi, and M. Ikeda, Electron. Lett. 32, 552 (1996).

${ }^{2}$ A. Ishibashi, J. Cryst. Growth 159, 555 (1996); K. Nakano and A. Ishibani (private communication); Workshop on the Physics and Chemistry of II-VI Semiconductors, Las Vegas, NV, 1996 (unpublished).

${ }^{3}$ I. S. Hauksson, J. Simpson, S. Y. Wang, K. A. Prior, and B. C. Cavenett, Appl. Phys. Lett. 61, 2208 (1992).

${ }^{4}$ B. N. Murdin, B. C. Cavenett, C. R. Pidgeon, J. Simpson, I. Hauksson, and K. A. Prior, Appl. Phys. Lett. 63, 2411 (1993).

${ }^{5}$ T. A. Kennedy, E. R. Glaser, B. N. Murdin, C. R. Pidgeon, K. A. Prior, and B. C. Cavenett, Appl. Phys. Lett. 65, 1112 (1994).

${ }^{6}$ T. Yao, T. Matsumoto, S. Sasaki, C. K. Chung, Z. Zhu, and F. Nishiyama, J. Cryst. Growth 138, 290 (1994).

${ }^{7}$ S. D. Setzler, M. Moldovan, Yu. Zhonghai, T. H. Myers, N. C. Giles, and L. E. Halliburton, Appl. Phys. Lett. 70, 2274 (1997).

${ }^{8}$ D. B. Laks, C. G. Van-de-Walle, G. F. Neumark, and S. T. Pantelides, Phys. Rev. Lett. 66, 648 (1991).

${ }^{9}$ D. B. Laks, C. G. Van-de-Walle, G. F. Neumark, P. E. Blochl, and S. T. Pantelides, Phys. Rev. B 45, 10965 (1992).

${ }^{10}$ A. Garcia and J. E. Northrup, Phys. Rev. Lett. 74, 113 (1995).

${ }^{11} \mathrm{~S}$. Little and B. R. Cooper, Talk E13.4 Meeting of Materials Research Society, Boston, 1996 (unpublished); Abstract N26.4 [Bull. Am. Phys. Soc. 42, 638 (1997)].

${ }^{12}$ S.-H. Wei and A. Zunger, Phys. Rev. B 37, 8958 (1988).

${ }^{13}$ Gun-Do-Lee, M. H. Lee, and Jisoon-Ihm, Phys. Rev. B 52, 1459 (1995).
${ }^{14}$ C. G. Van-de-Walle, D. B. Laks, G. F. Neumark, and S. T. Pantelides, Phys. Rev. B 47, 9425 (1993).

${ }^{15}$ Byoung-Ho-Cheong, C. H. Park, and K. J. Chang, Phys. Rev. B 51, 10610 (1995).

${ }^{16}$ C. H. Park and D. J. Chadi, Phys. Rev. Lett. 75, 1134 (1995).

${ }^{17}$ S. Pöykkö, M. J. Puska, T. Korhonen, and R. M. Nieminen, Mater. Sci. Eng., B B43, 1 (1997).

${ }^{18}$ S. Pöykkö, M. J. Puska, and R. M. Nieminen, Phys. Rev. B 57, 12 174 (1998).

${ }^{19}$ S. Gundel, D. Albert, J. Nürnberger, and W. Faschinger, Phys. Rev. B 60, R16271 (1999).

${ }^{20}$ S. G. Louie, S. Froyen, and M. L. Cohen, Phys. Rev: B 26, 1738 (1982).

${ }^{21}$ J. M. Wills (unpublished); D. L. Price and B. R. Cooper, Phys. Rev. B 39, 4945 (1989); D. L. Price, J. M. Wills, and B. R. Cooper, ibid. 46, 11368 (1992).

${ }^{22} \mathrm{~J}$. M. Wills, O. Eriksson, M. Alouani, and D. L. Price, Electronic Structure and Physical Properties of Solids, edited by H. Dreysse (Springer-Verlag, Berlin, 2000), pp. 148-167.

${ }^{23}$ C. S. Wang and B. M. Klein, Phys. Rev. B 24, 3393 (1981).

${ }^{24}$ M. Methfessel, Phys. Rev. B 38, 1537 (1988).

${ }^{25}$ D. Y. Jeon, H. P. Gislason, and G. D. Watkins, Phys. Rev. B 48, 7872 (1993).

${ }^{26}$ J. Petruzzello, J. Gaines, P. Van-Der-Sluis, D. Olego, and C. Ponzoni, Appl. Phys. Lett. 62, 1496 (1993).

${ }^{27}$ R. W. G. Wyckoff, Crystal Structures, 2nd ed. (Interscience Publishers, New York, 1964), Vol. 2, p. 5. 